\title{
Capecitabine compared with observation in resected biliary tract cancer (BILCAP): a randomised, controlled, multicentre, phase 3 study
}

John N Primrose ${ }^{a}$, Richard P Fox ${ }^{c}$, Daniel H Palmer ${ }^{d}$, Hassan Z Malike, Raj Prasad ${ }^{\dagger}$, Darius Mirza ${ }^{\mathrm{h}, \mathrm{i}}$, Alan Anthony', Pippa Corriek ${ }^{k}$ Stephen Falk', Meg Finch-Jones', Harpreet Wasanm, Paul Ross ${ }^{n}$, Lucy Wallo, Jonathan Wadsleyp , Jeff T R Evans ${ }^{q}$, Deborah Stockeng, Raaj Praseedomr, Yuk Ting Ma ${ }^{h}$, Brian Davidsons, John P Neoptolemos', Tim Iveson ${ }^{u}$, James Raftery ${ }^{b}$, Shihua Zhu ${ }^{b}$, David Cunninghamv, O James Gardenw, Clive Stubbs ${ }^{x}$, Juan W Valley, John Bridgewater ${ }^{z}$, on behalf of the BILCAP study group

\section{BILCAP study group}

JN Primrose, RP Fox, H Morement, O Chan, C Rees, YT Ma, T Hickish, S Falk, M Finch-Jones, I Pope, P Corrie, T Crosby, S Sothi, K Sharkland, D Adamson, L Wall, J Evans, J Dent, U Hombaiah, C Iwuji, A Anthoney, J Bridgewater, D Cunningham, R Gillmore, P Ross, S Slater, H Wasan, J Waters, JW Valle, D Palmer, H Malik, J Neoptolemos, O Faluyi, K Sumpter, U Dernedde, S Maduhusudan, G Cogill, C Archer, T Iveson, J Wadsley, S Darby, M Peterson, AA Mukhtar, JG Thorpe, A Bateman, D Tsang, S Cummins, L Nolan, E Beaumont, R Prasad, D Mirza, D Stocken, R Praseedom, B Davidson, J Raftery, S Zhu, J Garden, C Stubbs, F Coxon

a. Department of Surgery, University of Southampton, Southampton, UK

b. Health Economics Analysis Team, University of Southampton, Southampton, UK

c. Cancer Research UK Clinical Trials Unit, University of Birmingham, Birmingham, UK

d. University of Liverpool and Clatterbridge Cancer Centre, Liverpool, UK

e. Aintree University Hospital, Liverpool, UK

f. Department of Surgery, University of Leeds, Leeds, UK

g. Faculty of Medicine and Health, University of Leeds, Leeds, UK

h. University Hospitals Birmingham NHS Foundation Trust, Birmingham, UK

i. Women's Children's Hospital NHS Foundation Trust, Birmingham, UK

j. Leeds Teaching Hospitals NHS Trust, Leeds, UK

k. Cambridge University Hospitals NHS Foundation Trust, Cambridge, UK

I. University of Bristol Hospitals NHS Foundation Trust, Bristol, UK

m. Imperial Healthcare NHS Trust, London, UK 
n. Guys and St Thomas' NHS Foundation Trust, London, UK

o. Lothian University Hospitals NHS Trust, Edinburgh, UK

p. Weston Park Hospital, Sheffield, UK

q. Department of Oncology, University of Glasgow, Glasgow, UK

r. Addenbrookes Hospital, Cambridge, UK

s. Department of General Surgery, University College London Hospital, London, UK

t. Department of Surgery, University of Heidelberg, Heidelberg, Germany

u. University Hospitals Southampton, Southampton, UK

v. Royal Marsden NHS Trust, London, UK

w. Department of Medicine, University of Edinburgh, Edinburgh, UK

x. Birmingham Clinical Trials Unit, University of Birmingham, Birmingham, UK

y. University of Manchester and The Christie NHS Foundation Trust, Manchester, UK

z. UCL Cancer Institute, University College London, London, UK

Correspondence to:

Prof John Bridgewater, UCL Cancer Institute, University College London, London WC1E 6AG, UK j.bridgewater@ucl.ac.uk

or

Prof John N Primrose, Department of Surgery, Southampton General Hospital, Southampton SO16 6YD, UK j.n.primrose@soton.ac.uk 


\section{Research in context}

\section{Evidence before this study}

We searched PubMed, American Society of Clinical Oncology abstracts, and European Society for Medical Oncology abstracts for research articles published in English using the search terms "biliary tract cancer", "cholangiocarcinoma", "gall bladder cancer", "capecitabine", and "adjuvant". No date restrictions were applied. Biliary tract cancer is an uncommon cancer for which surgery is the only potentially curative treatment. We found that extant studies have either been statistically underpowered or inadequately designed to demonstrate a benefit for adjuvant systemic therapy.

\section{Added value of this study}

This randomised, controlled, multicentre, phase 3 study compared capecitabine with observation following resection of biliary tract cancer (BILCAP). Although the trial was negative for the prespecified primary endpoint (overall survival by intention to treat), the data taken as a whole strongly suggest a benefit of adjuvant capecitabine. The study shows that adjuvant capecitabine following surgery with curative intent for patient with biliary tract cancer improves overall survival compared with observation in the per-protocol population, with a clinically meaningful effect size of 14.7 months. Ongoing exploratory and translational analyses of BILCAP will help us to understand both the natural history and the impact of this cancer of unmet need.

\section{Implications of all the available evidence}

We believe this study is the first dedicated and sufficiently powered adjuvant study in biliary tract cancer and, as such, is uniquely placed to define the standard of care as capecitabine. It is unlikely that another study comparing treatment with surveillance will be done, although we await the results of the completed ASCOT study, a Japanese Clinical Oncology Group study comparing surveillance with S-1 chemotherapy. 


\section{Summary \\ Background}

Despite improvements in multidisciplinary management, patients with biliary tract cancer have a poor outcome. Only $20 \%$ of patients are eligible for surgical resection with curative intent, with 5 -year overall survival of less than $10 \%$ for all patients. To our knowledge, no studies have described a benefit of adjuvant therapy. We aimed to determine whether adjuvant capecitabine improved overall survival compared with observation following surgery for biliary tract cancer.

\section{Methods}

This randomised, controlled, multicentre, phase 3 study was done across 44 specialist hepatopancreatobiliary centres in the UK. Eligible patients were aged 18 years or older and had histologically confirmed cholangiocarcinoma or muscle-invasive gallbladder cancer who had undergone a macroscopically complete resection (which includes liver resection, pancreatic resection, or, less commonly, both) with curative intent, and an Eastern Cooperative Oncology Group performance status of less than 2. Patients who had not completely recovered from previous surgery or who had previous chemotherapy or radiotherapy for biliary tract cancer were also excluded. Patients were randomly assigned 1:1 to receive oral capecitabine (1250 mg/m² twice daily on days 1-14 of a 21-day cycle, for eight cycles) or observation commencing within 16 weeks of surgery. Treatment was not masked, and allocation concealment was achieved with a computerised minimisation algorithm that stratified patients by surgical centre, site of disease, resection status, and performance status. The primary outcome was overall survival. As prespecified, analyses were done by intention to treat and per protocol. This study is registered with EudraCT, number 2005-003318-13.

\section{Findings}

Between March 15, 2006, and Dec 4, 2014, 447 patients were enrolled; 223 patients with biliary tract cancer resected with curative intent were randomly assigned to the capecitabine group and 224 to the observation group. The data cutoff for this analysis was March 6, 2017. The median follow-up for all patients was 60 months (IQR 37-60). In the intention-to-treat analysis, median overall survival was 51.1 months (95\% CI $34 \cdot 6-59 \cdot 1)$ in the capecitabine group compared with $36 \cdot 4$ months $(29 \cdot 7-44 \cdot 5)$ in the observation group (adjusted hazard ratio [HR] 0.81,95\% $\mathrm{Cl} 0.63-1.04 ; \mathrm{p}=0.097$ ). In a protocol-specified sensitivity analysis, 
adjusting for minimisation factors and nodal status, grade, and gender, the overall survival HR was 0.71 $(95 \% \mathrm{Cl} 0.55-0.92 ; \mathrm{p}=0.010)$. In the prespecified per-protocol analysis (210 patients in the capecitabine group and 220 in the observation group), median overall survival was 53 months ( $95 \% \mathrm{Cl} 40$ to not reached) in the capecitabine group and 36 months (30-44) in the observation group (adjusted $\mathrm{HR} 0 \cdot 75,95 \% \mathrm{Cl} 0.58-$ $0.97 ; p=0.028)$. In the intention-to-treat analysis, median recurrence-free survival was 24.4 months $(95 \% \mathrm{Cl}$ 18.6-35.9) in the capecitabine group and 17.5 months (12.0-23.8) in the observation group. In the perprotocol analysis, median recurrence-free survival was 25.9 months (95\% $\mathrm{Cl} 19 \cdot 8-46 \cdot 3)$ in the capecitabine group and 17.4 months (12.0-23.7) in the observation group. Adverse events were measured in the capecitabine group only, and of the 213 patients who received at least one cycle, 94 (44\%) had at least one grade 3 toxicity, the most frequent of which were hand-foot syndrome in 43 (20\%) patients, diarrhoea in 16 (8\%) patients, and fatigue in $16(8 \%)$ patients. One $(<1 \%)$ patient had grade 4 cardiac ischaemia or infarction. Serious adverse events were observed in 47 (21\%) of 223 patients in the capecitabine group and $22(10 \%)$ of 224 patients in the observation group. No deaths were deemed to be treatment related.

\section{Interpretation}

Although this study did not meet its primary endpoint of improving overall survival in the intention-to- treat population, the prespecified sensitivity and per-protocol analyses suggest that capecitabine can improve overall survival in patients with resected biliary tract cancer when used as adjuvant chemotherapy following surgery and could be considered as standard of care. Furthermore, the safety profile is manageable, supporting the use of capecitabine in this setting.

\section{Funding}

Cancer Research UK and Roche. 


\section{Introduction}

Biliary tract cancer is an uncommon cancer in high-income countries. There are approximately 1200 and 9000 new cases per year in the UK and the USA, respectively. ${ }^{1,2}$ The incidence of biliary tract cancer is increasing, perhaps associated with an increasing incidence of gallstone disease. Potentially curative resection is feasible in $20 \%$ of presenting patients, ${ }^{3}$ and increasing centralisation of often complex surgery in specialist hepatopancreatobiliary centres aims to improve outcomes. ${ }^{4,5}$ The postoperative median overall survival is reported to be 18-30 months, with patients with positive lymph nodes and positive resection margins having a worse prognosis. ${ }^{6}$

The standard of care for patients with unresectable biliary tract cancer has been established as cisplatin and gemcitabine, suggesting that biliary tract cancers are chemosensitive malignancies. ${ }^{7,8}$ However, the value of adjuvant chemotherapy has not been investigated in a dedicated randomised trial. A subgroup of the ESPAC-3 trial $^{9}$ comprising 96 patients with biliary tract cancer and the study by Takada and colleagues, including 133 patients with non-curative biliary tract cancer resections, ${ }^{10}$ were not sufficiently statistically powered to define a standard of care. More recently, a randomised study ${ }^{11}$ of gemcitabine compared with surveillance in 225 patients with extrahepatic and perihilar cholangiocarcinoma resected with curative intent showed no difference in overall survival between the groups (hazard ratio [HR] 1·01, 95\% Cl 0.70-1·44; $\mathrm{p}=0.97)$. In addition, a phase 3 trial $^{12}$ testing adjuvant oxaliplatin plus gemcitabine compared with surveillance has recently been reported. Overall survival was not significantly different between the treatment groups (HR 1.08, 95\% Cl 0.70-1.66; p=0.74); however, a large effect size was seen (overall survival of $50 \cdot 8$ months in the surveillance group vs 75.8 months in the oxaliplatin plus gemcitabine group; HR 1.08, 95\% Cl.70-1.66). ${ }^{12}$ A meta-analysis ${ }^{13}$ of mostly non-randomised series has suggested the potential benefit for chemo- therapy as adjuvant therapy in patients with biliary tract cancer and node-positive disease, and of radiation-based adjuvant therapy in resection margin-positive (R1) subgroups, but given the quality of the data included in the analysis, these are still unproven hypotheses.

Capecitabine is an oral fluoropyrimidine prodrug that is effective as adjuvant chemotherapy treatment, either alone or in combination, in colorectal, ${ }^{14}$ oesophageal and gastric, ${ }^{15}$ and pancreatic ${ }^{9}$ malignancies. Fluoropyrimidines have evidence of activity in biliary tract cancer, ${ }^{10}$ are well tolerated, and used in everyday oncological practice. Although supportive clinical data are scarce, feasibility and compliance with treatment 
were considered crucial in this study and capecitabine was selected as protocol treatment. The BILCAP trial aimed to compare capecitabine with observation after resection of biliary tract cancer in specialist hepatopancreatobiliary centres in the UK.

\section{Methods}

\section{Study design and participants}

This randomised, controlled, multicentre, phase 3 study was done across 44 specialist hepatopancreatobiliary centres in the UK (appendix pp 4-6). Patients aged 18 years or older with histologically confirmed cholangiocarcinoma or muscle-invasive gallbladder cancer who had a macroscopically complete resection with curative intent were eligible. All patients should have had radical surgical treatment, which includes liver resection, pancreatic resection, or, less commonly, both. The Eastern Cooperative Oncology Group (ECOG) performance status had to be less than 2, and adequate renal, haematological, and liver function was required. Patients with pancreatic or ampullary cancer, mucosal gallbladder or unresolved biliary tree obstruction were ineligible. Patients who had not completely recovered from previous surgery or who had previous chemotherapy or radiotherapy for biliary tract cancer were also excluded. Criteria are described in full in the study protocol (appendix p 7).

Major protocol amendments included extending the start date of chemotherapy from 8 to 12 weeks from the date of definitive surgery on Oct 16,2007 , a further extension of study eligibility to 16 weeks after surgery on Sept 2, 2008, and the inclusion of extrahepatic cholangiocarcinoma following the completion of the ESPAC-3 study ${ }^{9}$ on Aug 26, 2008. These recommendations were made on the basis of the accumulating events during patient monitoring rather than in repeated interim analyses.

\section{Randomisation and masking}

Patients were randomly assigned 1:1 to the capecitabine group or the observation group. Treatment was not masked, and allocation concealment was achieved using a computerised minimisation algorithm that stratified patients by surgical centre, site of disease, resection status, and performance status. Concealment remained until the interventions were assigned by a central telephone-based randomisation service hosted by the Cancer Research UK Clinical Trials Unit (Birmingham). 


\section{Procedures}

Oral capecitabine $\left(1250 \mathrm{mg} / \mathrm{m}^{2}\right)$ was given post- operatively twice a day on days 1 to 14 of a 3-weekly cycle for 24 weeks (eight cycles), and observation commenced within 16 weeks of surgery. Following randomisation, chemotherapy was started as soon as possible after surgery and up to 12 weeks from surgery, with a maximum extension to 16 weeks from surgery. The protocol permitted dose modifications and cycle interruptions. In cases in which the capecitabine dose was reduced, it was not subsequently increased for any reason. In the case of dose interruptions due to toxicity for longer than 2 weeks, the patient was considered to be off treatment. There were no criteria for removal of patients from the study. Patients had the option to withdraw from trial treatment or follow-up at any stage. Furthermore, criteria for early treatment discontinuation, included safety concerns, patient deterioration, and administration of any other cancer treatment during the study treatment period. The full list of discontinuation criteria are in the protocol (appendix p 7).

All surgery was undertaken in specialist hepatopancreatobiliary centres, mandated in the UK. The surgical strategy was to achieve complete microscopic clearance of the disease, including liver or pancreatic resection. Patients with less than $1 \mathrm{~mm}$ clearance were classified as surgical margin-positive (R1) patients. Patients with intrahepatic cholangiocarcinoma underwent hepatectomy, and lymphadenectomy was not mandated for these patients. In the case of hilar cholangiocarcinoma, patients underwent hepatectomy, including segment 1 , along with radical excision of the extrahepatic biliary tree. Lymphadenectomy was done in accordance with local practice. Patients with muscle-invasive gallbladder cancer were treated by cholecystectomy when the gallbladder was in situ and hepatectomy, including the gallbladder bed. Excision of the extrahepatic biliary tree and the extent of lymphadenectomy was dependent on local practice. Biliary tract excision was commonly performed in patients in which the tumour involved the cystic duct. For tumours in the lower common bile duct, patients underwent pancreaticoduodenectomy (Whipple's procedure) with excision of the extrahepatic biliary tree and a standard lymphadenectomy.

Postoperative follow-up comprised CT scans every 6 months for the first 24 months and further CT scans at annual intervals with clinical review for up to 5 years. CT scans were done every 3 months in year 1 , every 6 months in year 2, and annually thereafter. Full blood count, biochemistry, and liver function tests were done at baseline, at the beginning of each treatment cycle for the capecitabine group, and every 3 months in 
year 1 and every 6 months in year 2 for all patients. Follow-up treatment for patients who had disease recurrence was not recorded. Toxicity was categorised according to the National Cancer Institute's Common Toxicity Criteria for Adverse Events, version 3.0. Toxicity was recorded continuously during treatment. Serious adverse events were monitored throughout.

Quality of life, recorded over 24 months at the same time as follow-up attendances, was measured using the European Organisation for the Research and Treatment of Cancer quality of life questionnaires QLQ-C30 (designed for all patients with cancer) and QLC-LMC21 (designed for patients with colorectal liver metastases). The EuroQoL Quality of Life Scale (EuroQoL-5D-5L) was recorded and used in the preplanned health economics analyses only.

This trial was run by the Cancer Research UK Clinical Trials Unit, University of Birmingham (UK), under the auspices of the UK National Cancer Research Institute Upper Gastrointestinal Cancer Studies Group and sponsored by the University of Southampton (UK). This trial was approved by the West Midlands MultiCentre Research Ethics Committee (05/MRE07/62), and all necessary regulatory approvals were obtained. All patients were required to give written informed consent, and the trial was done in accordance with the Declaration of Helsinki and the CONSORT and Consolidated Health Economic Evaluation Reporting Standards16 guidelines.

\section{Outcomes}

The primary outcome was overall survival, defined as the time from randomisation until the date of death or last date of follow-up for surviving patients. Prespecified secondary outcomes included a per-protocol analysis of outcomes, recurrence-free survival, toxicity, health economics, and quality of life. Recurrence-free survival was defined as the time from randomisation until the date of disease recurrence, death from disease, or date of last follow-up. Long-term outcome measures will be reported elsewhere once all surviving patients have a minimum follow-up of 60 months.

\section{Statistical analysis}

The initial sample size calculation was based on the assumption that the 24-month overall survival would be $20 \%$ in the observation group, ${ }^{5}$ and that treatment with capecitabine would improve this outcome by $12 \%$, 
from $20 \%$ to $32 \%$. As such, 360 patients and 270 events were needed to detect a HR of $0 \cdot 71$, with a twosided significance level of $5 \%$ and $80 \%$ power. The independent data monitoring committee (IDMC) met annually to review safety data and trial progress; no formal interim analyses were done. During the IDMC meeting of July, 2013 (at which point 364 patients had been recruited), it became clear that the observed number of events was less than originally estimated. Therefore, the IDMC recommended that the final analyses be done once 234 events had accrued. This number permitted detection of an increase in overall survival from $60 \%$ to $71 \%$ (HR 0.69 ), a marginally larger effect than originally planned. The IDMC instructed that screening cease in September, 2014, and recruitment in December, 2014. Analyses were done once the protocol- specified minimum follow-up period of 2 years was complete.

We did analyses according to the statistical analysis plan (appendix $\mathrm{p}$ 83). Primary analyses prespecified by protocol were by intention to treat, including all randomised patients. Analyses were also done per protocol, which excluded ineligible patients (appendix $\mathrm{p} 1$ ) and those failing to complete at least one cycle of capecitabine (prespecified in the statistical analysis plan). The safety population comprised any patient receiving at least one dose of capecitabine. Both groups were monitored for safety, and serious adverse event reporting was captured up to a maximum of 9 months from randomisation. With no specific intervention delivered, adverse events for toxicity were not monitored in the observation group.

We quantified overall and recurrence-free survival differences as HRs with 95\% Cls estimated using Cox proportional-hazards model with adjustment for minimisation factors. We did not adjust analyses by surgical centre because of the large number of participating centres $(n=44)$, leading to flat statistical modelling regions. Additionally, we did prespecified sensitivity analyses of overall survival and recurrence-free survival in the intention-to-treat population, adjusting the treatment effect for identified prognostic factors (appendix $p$ 2). We assessed the proportional hazards assumption for overall survival and recurrence-free survival by analysing Schoenfeld residuals, and time-varying effects were modelled when the assumption did not hold, with specification of time-varying effects guided by visual inspection of $-\log (-\log (S(t)))$ plots, where $S(t)$ is the survival probability at time $t$. We did preplanned subgroup analyses using adjusted Cox models, with heterogeneity tested via interaction terms. Subgroups were age (>60 vs $\leq 60$ years), sex, tumour size ( $>50$ vs $\leq 50 \mathrm{~mm}$ ), nodal status, tumour stage, disease grade, ECOG performance status, resection status, and site of disease. 
We assessed each quality-of-life domain by comparison of standardised area under the curve via a MannWhitney test. Economic analysis estimated incremental cost per quality-adjusted life-year (QALY), based on overall survival, and quality of life (EuroQoL-5D). Costs included intervention plus UK National Health Service use. Economic analyses were adjusted for baseline values, and we assessed uncertainty using costeffectiveness acceptability curves. We made no adjustment for multiplicity. The IDMC reviewed the data. No formal interim analyses were planned or done. All analyses were done in Stata, version 14. This study is registered with EudraCT, number 2005-003318-13.

\section{Role of the funding source}

The funder of the study had an advisory role in study design but no role in the running of the study, data collection, data analysis, data interpretation, or writing of the report. Upon completion of patient follow-up, JNP, RPF, CS, and JB had full access to all the data and the corresponding authors had final responsibility for the decision to submit for publication.

\section{Results}

Between March 15, 2006, and Dec 4, 2014, 447 patients (intention-to-treat population) were enrolled and randomly assigned to the capecitabine group $(n=223)$ or the observation group $(n=224$; figure 1$)$. The perprotocol population comprised 430 patients (210 in the capecitabine group and 220 in the observation group) following the exclusion of 17 patients, comprising seven (2\%) patients (three in the capecitabine group and four in the observation group) who were found to be ineligible after randomisation (appendix $p$ 1), nine (2\%) patients who did not receive capecitabine, and one $(<1 \%)$ patient was ineligible and also received no drug (appendix $\mathrm{p}$ 1). The required minimum follow-up of 24 months was reached in January, 2017, when the median follow-up for all patients was 60 months (IQR 37-60).

Baseline characteristics were well balanced between the two groups (table 1). The median time from surgery to randomisation was $10 \cdot 3$ weeks (IQR 8.4-12.1) in the capecitabine group and 10.4 weeks (9.0-12.1) in the observation group.

At the time of the final analysis (March 6, 2017), 114 (51\%) patients had died in the capecitabine group and 131 (58\%) patients had died in the observation group. Of these deaths, 241 (98\%) were related to biliary 
tract cancer (112 in the capecitabine group and 129 in the observation group), two (1\%) were due to unknown reasons (both in the capecitabine group), and two (1\%) resulted from other causes (both in the observation group; appendix $\mathrm{p}$ 1). In the intention-to-treat analysis, median overall survival was 51.1 months $(95 \% \mathrm{Cl} 34.6-59 \cdot 1)$ in the capecitabine group and 36.4 months (29.7-44.5) in the observation group (HR $0.81,95 \% \mathrm{Cl} 0.63-1 \cdot 04 ; \mathrm{p}=0.097$; figure 2), when adjusted for minimisation factors other than surgical centre. Planned sensitivity analyses in the intention-to-treat population explored the effect of identified prognostic factors (nodal status, grade of disease, and sex). Adjusting for these and minimisation factors resulted in an overall survival HR of $0.71(95 \% \mathrm{Cl} 0.55-0.92 ; \mathrm{p}=0.010)$. In the per-protocol analysis (figure 2), median overall survival was 53 months ( $95 \% \mathrm{Cl} 40$ to not reached) in the capecitabine group and 36 months (30-44) in the observation group (adjusted HR 0.75, 95\% Cl 0.58-0.97; $p=0.028$ ).

$280(63 \%)$ of 447 patients had disease recurrence (134 [60\%] of 223 patients in the capecitabine group and 146 [65\%] of 224 patients in the observation group). In the intention-to-treat analysis, median recurrence-free survival was 24.4 months $(95 \% \mathrm{Cl} 18.6-35.9)$ in the capecitabine group and 17.5 months $(12 \cdot 0-23.8)$ in the observation group (figure 3). The relative difference in risk between treatment groups differed over time and, as such, Cox models with time-varying effects were fitted. The adjusted recurrence-free survival HR was $0.75(95 \% \mathrm{Cl} 0.58-0.98 ; \mathrm{p}=0.033)$ in the first 24 months from randomisation, with no evidence of a difference in the period from 24 to 60 months (recurrence-free survival HR 1.48, 95\% $\mathrm{Cl} 0 \cdot 80-2 \cdot 77 ; \mathrm{p}=0 \cdot 21$ ). In the per- protocol analysis, median recurrence-free survival was 25.9 months (95\% $\mathrm{Cl} 19 \cdot 8-46 \cdot 3)$ in the capecitabine group and 17.4 months (12.0-23.7) in the observation group (figure 3 ). The adjusted recurrence-free survival HR from 0 to 24 months was $0.70(95 \% \mathrm{Cl} 0.54-0.92 ; \mathrm{p}=0.0093)$, and there was no evidence of a difference beyond 24 months (recurrence-free survival HR 1.55, 95\% Cl 0.82-2.93; $p=0 \cdot 18$; see appendix pp 1-3 for final overall survival and recurrence-free survival models).

The median capecitabine dose was $1250 \cdot 0 \mathrm{mg} / \mathrm{m}^{2}$ twice daily (IQR 1060.9-1250.0). All but ten (4\%) patients who started capecitabine received at least one cycle of capecitabine, and 122 (55\%) patients completed eight cycles of capecitabine. Of the 213 patients who started treatment, $99(46 \%)$ had at least one dose reduction. Of the 69 (32\%) who discontinued treatment because of toxicity, the most common complaints were hand-foot syndrome in ten patients (14\%), diarrhoea in nine patients (13\%), and other (patients could cite more than one toxicity type) in 21 (31\%) patients. 
Adverse events were only recorded in the capecitabine group, and serious adverse events were recorded in both groups. Treatment toxicity was assessed in the safety population (213 patients in the capecitabine group), and 212 patients reported 4694 toxicities. The grade was unknown in 21 (<1\%) events. Of the 213 patients, $94(44 \%)$ had at least one grade 3 toxicity, and one patient $(<1 \%)$ had grade 4 cardiac ischaemia or infarction (table 2). The most frequent grade 3 events were hand-foot syndrome in 43 (20\%) of 213 patients, diarrhoea in $16(8 \%)$ patients, or fatigue in $16(8 \%)$ patients. Serious adverse events were observed in 47 (21\%) of 223 patients (64 events) in the capecitabine group and 22 (10\%) of 224 patients (29 events) in the observation group. Of the 64 serious adverse events in the capecitabine group, 33 (52\%) were related to treatment and, of those, five (8\%) were cardiac events related to capecitabine (table 3). None of the serious adverse events in the capecitabine group resulted in death, and three (10\%) of those reported in the observation group resulted in death (appendix $\mathrm{p} 4$ ).

Prespecified subgroup analyses of clinical factors are presented in the forest plot (figure 4). In the intentionto- treat population, benefit of capecitabine was indicated in men (HR $0 \cdot 70,95 \% \mathrm{Cl} 0 \cdot 50-0.99)$ and those with poorly differentiated disease $(0.60,0.39-0.93)$. There was no statistical evidence of heterogeneity. 1915 quality-of-life questionnaires were returned by 433 (97\%) of 447 patients (216 [97\%] of 223 in the capecitabine group and 217 [97\%] of 224 in the observation group). Area under the curve was standardised by time, and hence the standardised area under the curve is interpreted as the average monthly quality of life. The full set of results is provided in table 4. Statistically significant differences were observed in the social functioning scale of the QLQ-C30, with a median standardised area under the curve of 76.2 (IQR $56 \cdot 9-91 \cdot 7)$ in the capecitabine group and $83.3(64 \cdot 6-95 \cdot 8)$ in the observation group ( $p=0.0060)$. Analyses of QLQ-LMC-21 identified increased taste symptoms in the capecitabine group $(p=0.042)$, with a median standardised area under the curve of 0.0 (IQR 0.0-11.1) in the capecitabine group and $0.0(0 \cdot 0-6 \cdot 3)$ in the observation group, and peripheral neuropathy $(p=0.0016)$ with a median standardised area under the curve of $0.0(0.0-13.5)$ in the capecitabine group and $0.0(0.0-4 \cdot 2)$ in the observation group, although peripheral neuropathy should be interpreted as hand-foot syndrome. These statistical differences in quality of life are unlikely to have translated into clinical significance. No other statistically significant differences were observed (table 4). 
The mean QALY gain at 2 years was $0.035(95 \% \mathrm{Cl}-0.034$ to 0.104$)$, leading to an incremental cost per QALY of just under $£ 13300$ (US\$17 200). Linear extrapolation to 5 years reduced the incremental cost per QALY to $£ 2725$ (\$3538). The cost effectiveness accept- ability curve indicated a probability of more than $90 \%$ of capecitabine being cost-effective at willingness to pay more than $£ 18000$ ( $\$ 23377$; appendix p 4).

\section{Discussion}

The BILCAP study, which compared capecitabine with observation as an adjuvant in biliary tract cancer resected with curative intent, provides evidence that capecitabine can improve overall survival. Although the overall survival primary endpoint analysed in the intention-to- treat population did not reach statistical significance, the sensitivity analyses of this population, the per-protocol overall survival and recurrence-free survival analyses showed benefit, and the overall survival effect size of 14.7 months is clinically meaningful. The intention-to- treat (statistically negative) and per-protocol (statistically positive) populations differed by 17 patients who were either found to be ineligible (appendix $\mathrm{p} 1$ ) or were randomly assigned to but did not receive capecitabine. Of the patients who did not receive capecitabine, the most common reason cited was that the patient no longer wished to participate in the trial (appendix $\mathrm{p} 1$ ).

The intention-to-treat and per-protocol analyses revealed no evidence of a difference in recurrence-free survival in the period between 24 and 60 months, suggesting that deferred recurrence occurred in the capecitabine group. This finding will be explored in the long-term survival analyses to be reported once 5 years of follow-up has been met.

The limitations of this study include the long recruitment period of 10 years, during which time approaches to the clinical trial process have become more defined. An unintended consequence is that the protocol, which was acceptable when written in 2005 , can be criticised; for example, there was no fully defined statistical analysis plan when the study started, but it is mandatory in a 2019 study. Additionally, the heterogeneity of biliary tract cancers, both surgically and, more recently, biologically, makes an overall interpretation of our findings more complex. Furthermore, the surgical centre was not included in the modelling analyses adjusted for minimisation factors. 
Adverse events were modest, and the incidence of some potentially serious toxic effects such as fluoropyrimidine-related cardiac vasospasm was significantly less than seen in similar studies, perhaps because any serious cardiac comorbidity had been unmasked in preparation for and during hepatopancreatobiliary surgery. Although some significant changes in quality of life were observed, the differences were modest and support a tolerable and deliverable regimen that is cost-effective. Compliance to capecitabine in BILCAP was lower than for colorectal cancer ${ }^{14}$ but equivalent to that for patients who had undergone hepatopancreatobiliary surgery. ${ }^{9}$ Further analysis of dose intensity to determine any effect on outcome will be reported elsewhere.

We have reported median overall survival of more than 50 months following potentially curative surgery for biliary tract cancer, which suggests an improvement on historical controls that is likely to be a reflection of improved surgical selection and management, as well as the patient selection criteria (fitness) required for the study. Centralisation in the care of complex medicine has resulted in improved outcomes, ${ }^{5}$ specifically for cancer surgery, and has been the principle behind the establishment of specialist hepatopancreatobiliary centres in the UK. This improvement became apparent during recruitment and required a protocol amendment changing the observed 2-year survival in the observation group from $20 \%$ to $60 \%$. Additionally, during the recruitment period, the standard of care in advanced disease was established as cisplatin and gemcitabine, which might have affected the unanticipated improvement in overall survival.7,8

Biliary tract cancer is an uncommon cancer and, as reflected by the BILCAP study duration as well as the experience of other investigators, adjuvant studies are challenging. Although BILCAP was not a statistically positive study by the primary intention-to-treat analysis, the position of equipoise among oncologists might be sufficiently affected by the weight of the overall positive body of BILCAP data as to render a future study with an observation group unfeasible. We note that the control group in the current European adjuvant study ${ }^{17}$ has been changed to capecitabine from observation, perhaps for this reason. We believe that the body of BILCAP data as a whole is sufficient to propose a benefit for adjuvant capecitabine as a standard of care in the adjuvant management of biliary tract cancer resected with curative intent.

Biliary tract cancer is emerging as a biologically heterogeneous group of cancers, ${ }^{18}$ which perhaps explains the failure of targeted therapies in unselected patient populations to demonstrate benefit in advanced 
disease, ${ }^{19-21}$ although there is promise in selected populations..$^{22,23}$ The translational research outcomes for BILCAP are therefore crucial for the future testing of more effective therapies.

In summary, although the BILCAP study did not meet its primary endpoint of improving overall survival in the intention-to-treat population, the sensitivity and secondary analyses suggest that capecitabine can improve overall survival in resected biliary tract cancer when used as adjuvant chemotherapy following surgery and could be considered as standard of care. Furthermore, the safety profile is manageable and the quality of life data favourable, supporting the use of capecitabine in this setting.

\section{Contributors}

JNP and JB were responsible for the literature search, study design, data collection, data analysis, data interpretation, and writing. RPF did all statistical analyses. RPF, DS, CS, OJG, DC, and JWV were responsible for the study design, data collection, data interpretation, and writing. DHP, HZM, RajP, DM, AA, PC, SF, MF-J, HW, PR, LW, JW, JPN, RaajP, YTM, BD, and TI were responsible for the data collection, data interpretation, and writing. JR and SZ were responsible for the data interpretation and writing. A list of all contributing investigators is provided in the appendix pp 4-5.

\section{Declaration of interests}

JB has received honoraria, speakers' fees, and travel support from Roche, Amgen, Merck Serono, Servier, Celgene, and Merck Sharp \& Dohme.

DC has received research funding from Amgen, AstraZeneca, Bayer, Celgene, Merrimack/Medimmune, and Merck Serono. JTRE has received research funding and honoraria from Eisai, Clovis, Karus, Baxalta, Bayer, Celgene, GlaxoSmithKline, Otsuka, Roche, TC Biopharm, Immunova, Basilea, e-Therapeutics, Immunocore, Vertex, Verastem, Daiichi, Merck, and Bristol-Myers Squibb. TI reports honoraria and travel expenses from Lilly, Roche, Celgene, Bayer, and Servier.

YTM reports personal fees from Bayer and Baxalta. PR reports personal fees and non-financial support from Celgene, Bristol-Myers Squibb, Oncosil, Servier, Bayer, Sirtex, and Merck Serono. JWV reports personal fees from Lilly, AstraZeneca, Merck, Delcath, Agios, and Celgene; and personal fees and non-financial support from Ipsen, Novartis, Celgene, Eisai, Bayer, Sanofi-Genzyme, Sobi, Baxalta, Lilly, and AstraZeneca. 
All potential conflicts of interests were outside of the submitted work. All other authors declare no competing interests.

\section{Data sharing}

Data collected for the study, including individual participant data and a data dictionary defining each field in the set, will be made available to others by signed data access agreement.

\section{Acknowledgments}

This study is supported by Cancer Research UK and an unrestricted educational grant to support recruitment and translational sample collection from Roche. JB is supported by the University College London Hospitals and University College London Biomedical Research Centre (London, UK). DC is funded by the Royal Marsden National Institute for Health Biomedical Research Centre (London, UK). We thank the patients and their families, without whom this trial would not have been possible and the independent data monitoring committee: Chris Russell (chair), Peter Clark, and Roger A'Hern. We thank Alison Backen for technical assistance.

\section{References}

1 Patel T. Increasing incidence and mortality of primary intrahepatic cholangiocarcinoma in the United States. Hepatology 2001; 33: 1353-57.

2 Taylor-Robinson SD, Toledano MB, Arora S, et al. Increase in mortality rates from intrahepatic cholangiocarcinoma in England and Wales 1968-1998. Gut 2001; 48: 816-20.

3 Bridgewater J, Galle PR, Khan SA, et al. Guidelines for the diagnosis and management of intrahepatic cholangiocarcinoma. J Hepatol 2014; 60: 1268-89.

4 NICE. Pancreatic cancer in adults: diagnosis and management. 2018. nice.org.uk/guidance/ng85 (accessed March 3, 2019).

5 Birkmeyer JD, Stukel TA, Siewers AE, Goodney PP, Wennberg DE, Lucas FL. Surgeon volume and operative mortality in the United States. N Engl J Med 2003; 349: 2117-27.

6 DeOliveira ML, Cunningham SC, Cameron JL, et al. Cholangiocarcinoma: thirty-one-year experience with 564 patients at a single institution. Ann Surg 2007; 245: 755-62. 
Valle J, Wasan $\mathrm{H}$, Palmer $\mathrm{DH}$, et al. Cisplatin plus gemcitabine versus gemcitabine for biliary tract cancer. N Engl J Med 2010; 362: 1273-81.

8 Bridgewater J, Lopes A, Palmer D, et al. Quality of life, long-term survivors and long-term outcome from the ABC-02 study. Br J Cancer 2016; 114: 965-71.

9 Neoptolemos JP, Palmer DH, Ghaneh P, et al. Comparison of adjuvant gemcitabine and capecitabine with gemcitabine monotherapy in patients with resected pancreatic cancer (ESPAC-4): a multicentre, open-label, randomised, phase 3 trial. Lancet 2017; 389: 1011-24.

10 Takada T, Nimura Y, Katoh H, et al. Prospective randomized trial of 5-fluorouracil, doxorubicin, and mitomycin C for non-resectable pancreatic and biliary carcinoma: multicenter randomized trial. Hepatogastroenterology 1998; 45: 2020-26.

11 Ebata T, Hirano S, Konishi M, et al. Randomized clinical trial of adjuvant gemcitabine chemotherapy versus observation in resected bile duct cancer. Br J Surg 2018; 105: 192-202.

12 Edeline J, Bonnetain F, Phelip JM, et al. Gemox versus surveillance following surgery of localized biliary tract cancer: results of the PRODIGE 12-ACCORD 18 (UNICANCER GI) phase III trial. J Clin Oncol 2017; 35 (4 suppl): 225.

13 Horgan AM, Amir E, Walter T, Knox JJ. Adjuvant therapy in the treatment of biliary tract cancer: a systematic review and meta-analysis. J Clin Oncol 2012; 30: 1934-40.

14 Twelves C, Wong A, Nowacki MP, et al. Capecitabine as adjuvant treatment for stage III colon cancer. N Engl J Med 2005; 352: 2696-704.

15 Alderson D, Cunningham D, Nankivell M, et al. Neoadjuvant cisplatin and fluorouracil versus epirubicin, cisplatin, and capecitabine followed by resection in patients with oesophageal adenocarcinoma (UK MRC OE05): an open-label, randomised phase 3 trial. Lancet Oncol 2017; 18: 1249-60.

16 Husereau D, Drummond M, Petrou S, et al. Consolidated Health Economic Evaluation Reporting Standards (CHEERS) statement. Int J Technol Assess Health Care 2013; 29: 117-22.

17 Stein A, Arnold D, Bridgewater J, et al. Adjuvant chemotherapy with gemcitabine and cisplatin compared to observation after curative intent resection of cholangiocarcinoma and muscle invasive gallbladder carcinoma (ACTICCA-1 trial)—a randomized, multidisciplinary, multinational phase III trial. BMC Cancer 2015; 15: 564.

18 Jusakul A, Cutcutache I, Yong $\mathrm{CH}$, et al. Whole-genome and epigenomic landscapes of etiologically distinct subtypes of cholangiocarcinoma. Cancer Discov 2017; 7: 1116-35. 
19 Malka D, Cervera P, Foulon S, et al. Gemcitabine and oxaliplatin with or without cetuximab in advanced biliary-tract cancer (BINGO): a randomised, open-label, non-comparative phase 2 trial. Lancet Oncol 2014; 15: 819-28.

20 Moehler M, Maderer A, Schimanski C, et al. Gemcitabine plus sorafenib versus gemcitabine alone in advanced biliary tract cancer: a double-blind placebo-controlled multicentre phase II AIO study with biomarker and serum programme. Eur J Cancer 2014; 50: 3125-35.

21 Javle M, Churi C, Kang HC, et al. HER2/neu-directed therapy for biliary tract cancer. J Hematol Oncol 2015; 8: 58.

22 Lowery MA, Abou-Alfa GK, Burris HA, et al. Phase I study of AG-120, an IDH1 mutant enzyme inhibitor: Results from the cholangiocarcinoma dose escalation and expansion cohorts. J Clin Oncol 2017; 35 (15 suppl): 4015.

23 Goyal L, Saha SK, Liu LY, et al. Polyclonal secondary FGFR2 mutations drive acquired resistance to FGFR inhibition in patients with FGFR2 fusion-positive cholangiocarcinoma. Cancer Discov 2017; 7: 252-63. 


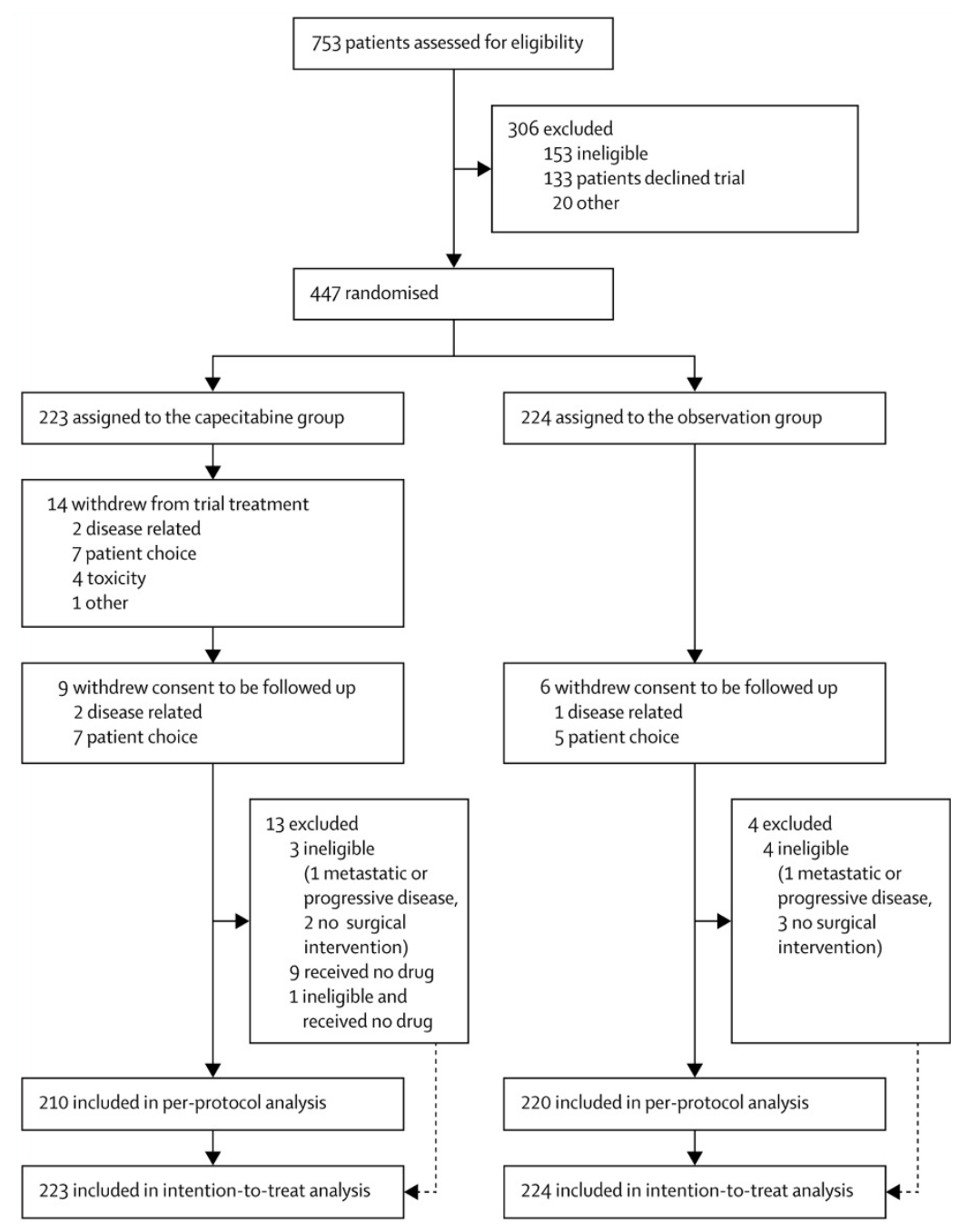

Figure 1: Trial profile 

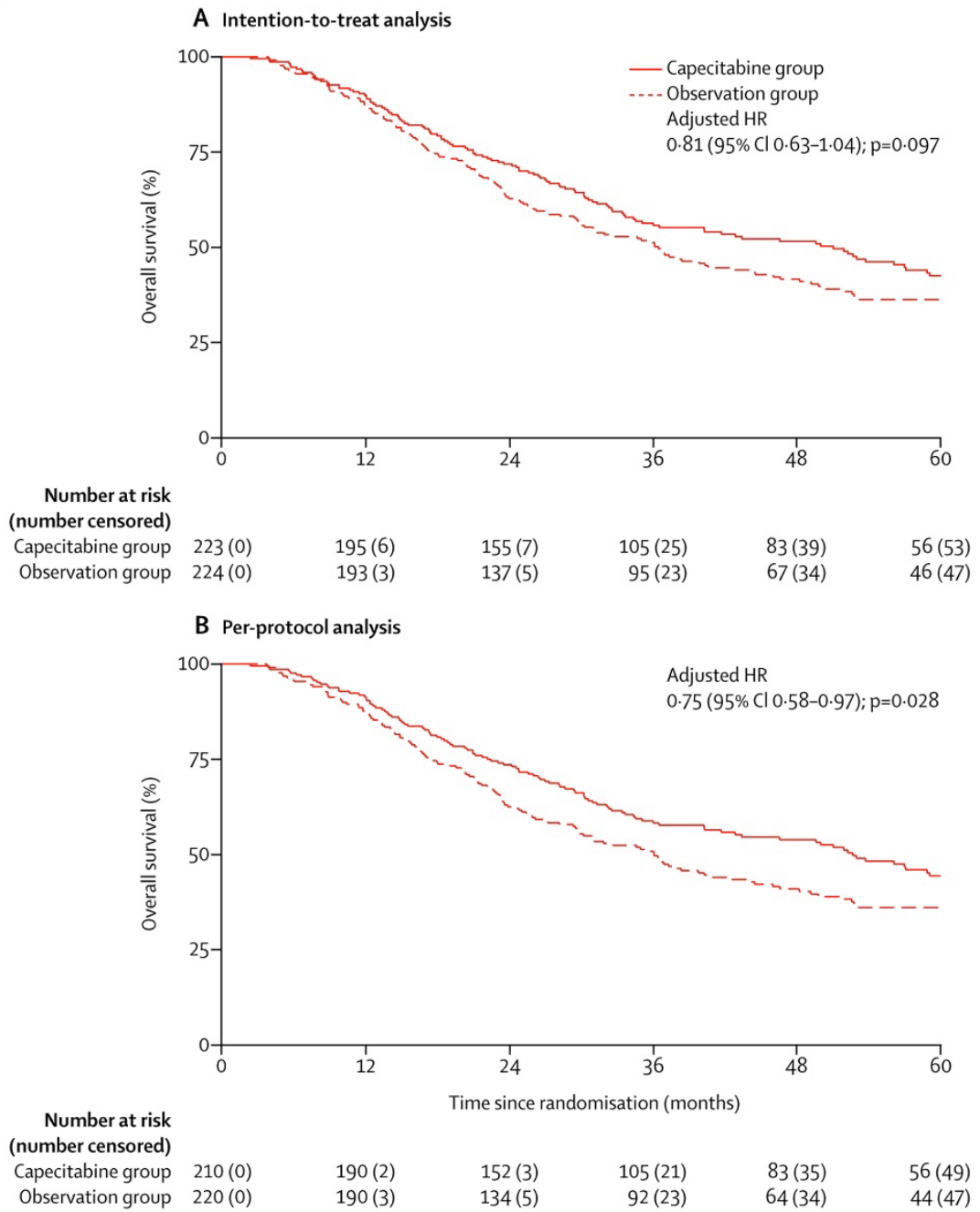

Figure 2: Overall survival by intention-to-treat $(A)$ and per-protocol $(B)$ analyses $\mathrm{HR}=$ hazard ratio. 


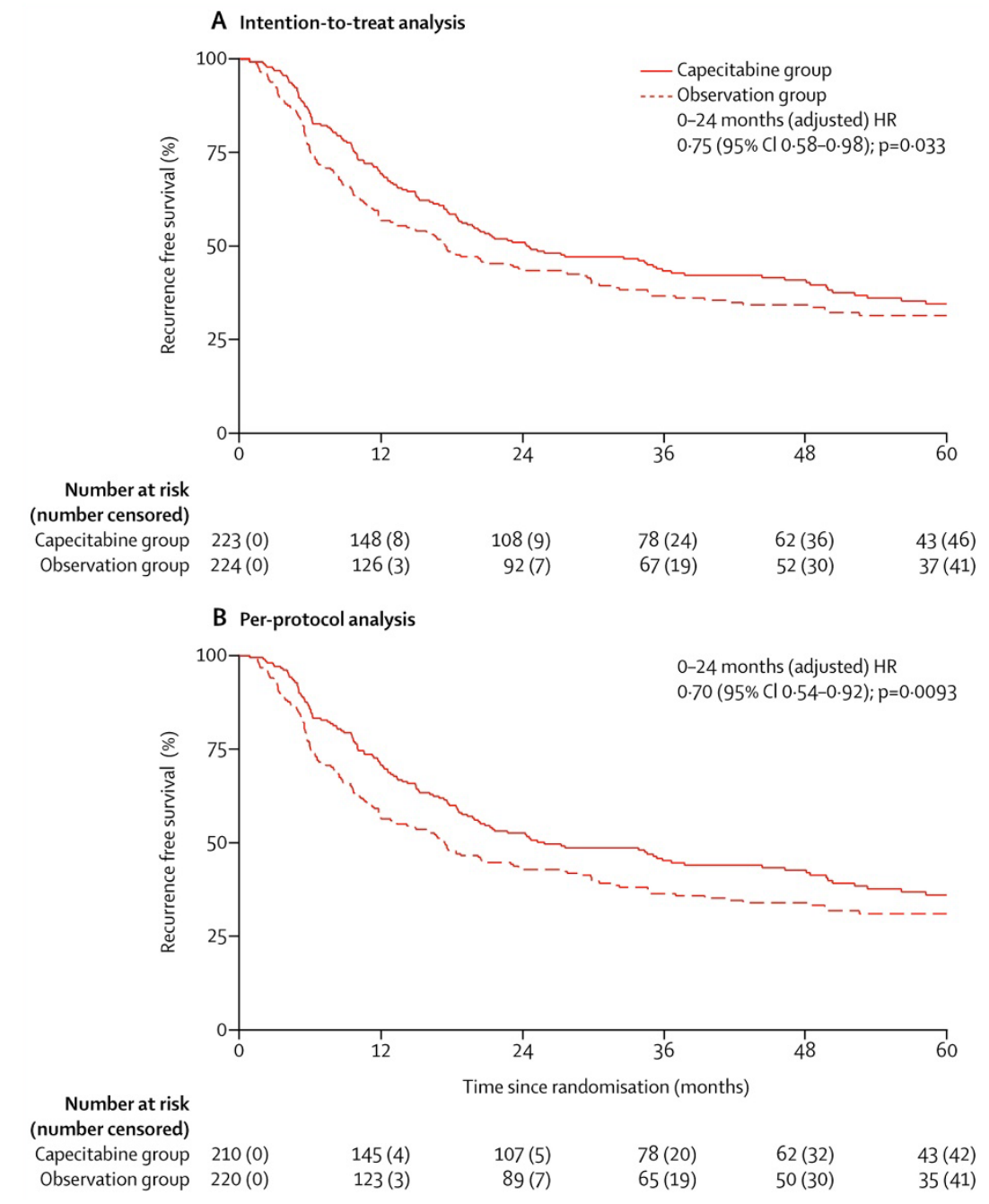

Figure 3: Recurrence-free survival by intention-to-treat (A) and per-protocol (B) analyses $\mathrm{HR}=$ hazard ratio 


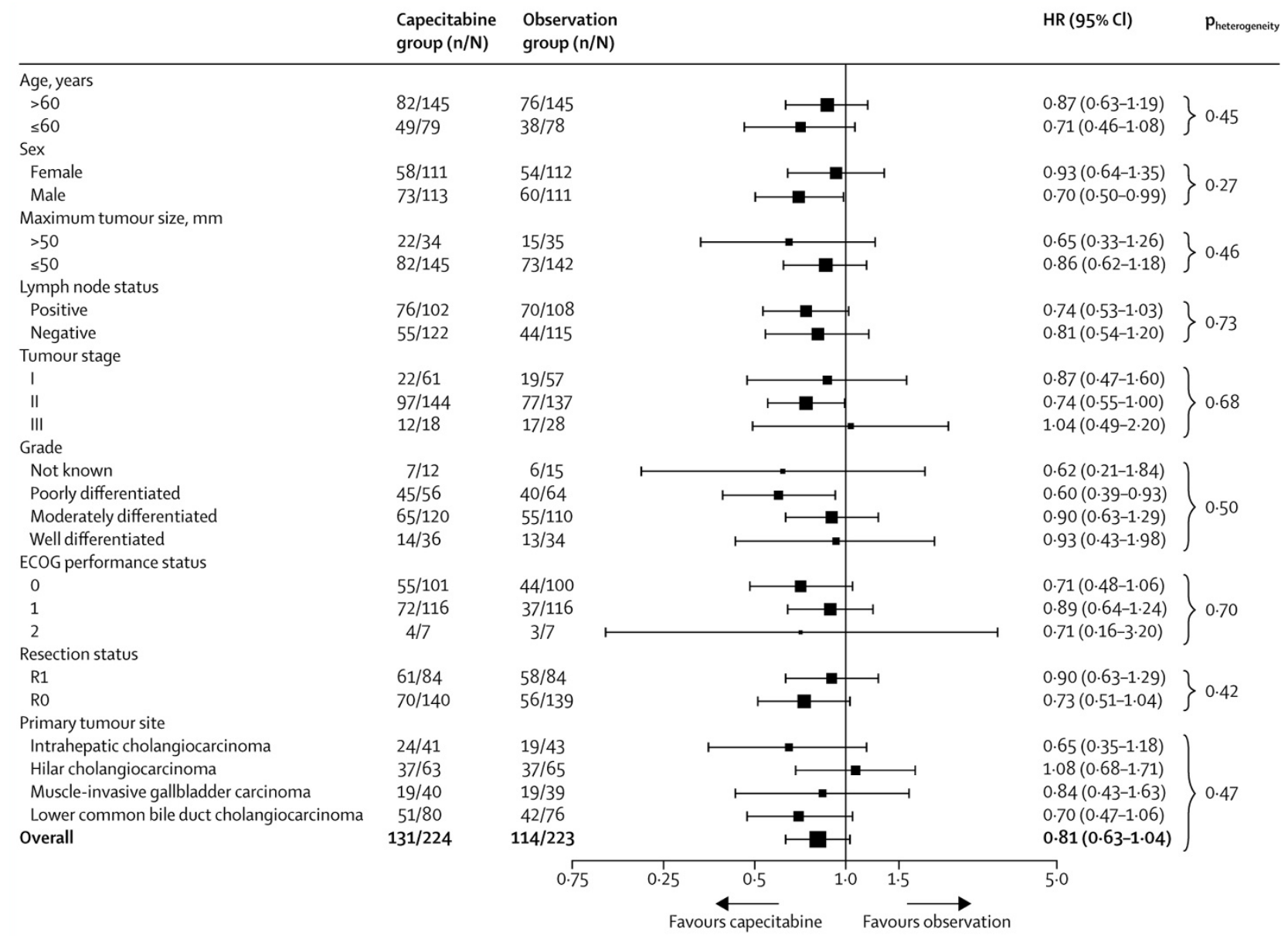

Figure 4: Subgroup analyses of overall survival in the intention-to-treat population Heterogeneity assessed through fitting of interactions terms in Cox survival models. ECOG=Eastern Cooperative Oncology Group. HR=hazard ratio. R0=negative resection margin. R1=positive resection margin. 


\begin{tabular}{|c|c|c|}
\hline & $\begin{array}{c}\text { Capecitabine group } \\
(n=223)\end{array}$ & $\begin{array}{l}\text { Observation group } \\
(\mathrm{n}=224)\end{array}$ \\
\hline \multicolumn{3}{|c|}{$x_{1}+2+2$} \\
\hline Female & $112(50 \%)$ & $111(50 \%)$ \\
\hline Male & $111(50 \%)$ & $113(50 \%)$ \\
\hline Age, years & $62(55-68)$ & $64(55-69)$ \\
\hline \multicolumn{3}{|l|}{ Primary tumour site } \\
\hline Intrahepatic cholangiocarcinoma & $43(19 \%)$ & $41(18 \%)$ \\
\hline Hilar cholangiocarcinoma & $65(29 \%)$ & $63(28 \%)$ \\
\hline Muscle-invasive gallbladder carcinoma & $39(17 \%)$ & $40(18 \%)$ \\
\hline Mucosal gallbladder carcinoma & 0 & 0 \\
\hline Lower common bile duct cholangiocarcinoma & $76(34 \%)$ & $80(36 \%)$ \\
\hline \multicolumn{3}{|l|}{ Resection status } \\
\hline RO & $139(62 \%)$ & $140(63 \%)$ \\
\hline R1 & $84(38 \%)$ & $84(38 \%)$ \\
\hline \multicolumn{3}{|l|}{ ECOG performance status } \\
\hline 0 & $100(45 \%)$ & $101(45 \%)$ \\
\hline 1 & $116(52 \%)$ & $116(52 \%)$ \\
\hline 2 & $7(3 \%)$ & $7(3 \%)$ \\
\hline \multicolumn{3}{|l|}{ Tumour stage } \\
\hline 1 & $57(26 \%)$ & $61(27 \%)$ \\
\hline II & $137(61 \%)$ & $144(64 \%)$ \\
\hline IIII & $28(13 \%)$ & $18(8 \%)$ \\
\hline IV & $1(<1 \%)$ & 0 \\
\hline Missing data & 0 & $1(<1 \%)$ \\
\hline \multicolumn{3}{|l|}{ Lymph node status } \\
\hline NO & $115(52 \%)$ & $121(54 \%)$ \\
\hline N1 & $108(48 \%)$ & $102(46 \%)$ \\
\hline Missing data & 0 & $1(<1 \%)$ \\
\hline \multicolumn{3}{|l|}{ Disease grade } \\
\hline Well differentiated & $34(15 \%)$ & $36(16 \%)$ \\
\hline Moderately differentiated & $110(49 \%)$ & $120(54 \%)$ \\
\hline Poorly differentiated & $64(29 \%)$ & $56(25 \%)$ \\
\hline Not determined & $12(5 \%)$ & $9(4 \%)$ \\
\hline Not known & $3(1 \%)$ & $2(1 \%)$ \\
\hline Missing data & 0 & $1(<1 \%)$ \\
\hline Haemoglobin $(\mathrm{g} / \mathrm{dL})$ & $12(12-13)$ & $13(12-14)$ \\
\hline White blood cell count, $\times 10^{9}$ cells per $\mathrm{L}$ & $7(6-8)$ & $7(6-8)$ \\
\hline Absolute neutrophil count, $\times 10^{9}$ cells per $\mathrm{L}$ & $4(3-5)$ & $4(3-5)$ \\
\hline Platelet count, $\times 10^{9}$ per $\mathrm{L}$ & $279(231-346)$ & $280(243-343)$ \\
\hline Glomerular filtration rate, $\mathrm{mL} / \mathrm{min}$ & $92(77-113)$ & $94(77-111)$ \\
\hline Aspartate aminotransferase, U/L & $27(22-35)$ & $27(20-38)$ \\
\hline Alanine aminotransferase, U/L & $27(20-41)$ & $26(18-40)$ \\
\hline Bilirubin, $\mu \mathrm{mol} / \mathrm{L}$ & $8(6-10)$ & $8(5-11)$ \\
\hline Creatinine, $\mu \mathrm{mol} / \mathrm{L}$ & $67(58-76)$ & $67(58-77)$ \\
\hline Tumour size, mm & $25(19-45)$ & $25(20-44)$ \\
\hline \multicolumn{3}{|l|}{ Resection type } \\
\hline Liver & $129(58 \%)$ & $124(55 \%)$ \\
\hline Pancreas & $92(41 \%)$ & $97(43 \%)$ \\
\hline Other & $2(1 \%)$ & $2(1 \%)$ \\
\hline Missing data & 0 & $1(<1 \%)$ \\
\hline
\end{tabular}

Data are $\mathrm{n}(\%)$ or median (IQR). N0=negative. $\mathrm{N} 1=$ positive. $\mathrm{R} 0=$ =negative resection margin. $\mathrm{R} 1=$ =positive resection margin. ECOG=Eastern Cooperative Oncology Group.

Table 1: Baseline characteristics 


\begin{tabular}{|l|c|c|}
\hline & Grade 1 and 2 & Grade 3 \\
\hline Hand-foot syndrome & $127(60 \%)$ & $43(20 \%)$ \\
\hline Fatigue & $159(75 \%)$ & $16(8 \%)$ \\
\hline Diarrhoea & $121(57 \%)$ & $16(8 \%)$ \\
\hline $\begin{array}{l}\text { Gastrointestinal or abdominal } \\
\text { pain not otherwise specified }\end{array}$ & $61(29 \%)$ & $10(5 \%)$ \\
\hline Neutrophils or granulocytes & $45(21 \%)$ & $4(2 \%)$ \\
\hline Bilirubin & $42(20 \%)$ & $3(1 \%)$ \\
\hline Nausea & $106(50 \%)$ & $2(1 \%)$ \\
\hline Oral mucositis or stomatitis & $94(44 \%)$ & $2(1 \%)$ \\
\hline $\begin{array}{l}\text { Skin rash or desquamation } \\
\text { (dermatology) }\end{array}$ & $31(15 \%)$ & $2(1 \%)$ \\
\hline $\begin{array}{l}\text { Insomnia (constitutional } \\
\text { symptoms) }\end{array}$ &.. & $2(1 \%)$ \\
\hline Gastrointestinal ascites &.. & $2(1 \%)$ \\
\hline Biliary sepsis &.. & $2(1 \%)$ \\
\hline Vomiting & $49(23 \%)$ & $1(<1 \%)$ \\
\hline Fever & $30(14 \%)$ & $1(<1 \%)$ \\
\hline Low platelet count & $25(12 \%)$ & $1(<1 \%)$ \\
\hline Dry skin (dermatology/skin) &.. & $1(<1 \%)$ \\
\hline Lip swelling (dermatology/skin) &.. & $1(<1 \%)$ \\
\hline Gastrointestinal dehydration &.. & $1(<1 \%)$ \\
\hline Gastrointestinal obstruction &.. & $1(<1 \%)$ \\
\hline Infection &.. & $1(<1 \%)$ \\
\hline Limb oedema (lymphatics) &.. & $1(<1 \%)$ \\
\hline $\begin{array}{l}\text { Alanine aminotransferase } \\
\text { (metabolic/laboratory) }\end{array}$ &.. & $1(<1 \%)$ \\
\hline $\begin{array}{l}\text { Aspartate aminotransferase } \\
\text { (metabolic/laboratory) }\end{array}$ &.. & $1(<1 \%)$ \\
\hline $\begin{array}{l}\text { Alkaline phosphatase } \\
\text { (metabolic/laboratory) }\end{array}$ &.. & $1(<1 \%)$ \\
\hline $\begin{array}{l}\text { Low serum potassium } \\
\text { (metabolic/laboratory) }\end{array}$ &.. & $1(<1 \%)$ \\
\hline $\begin{array}{l}\text { Y-glutamyltransferase } \\
\text { (metabolic/laboratory) }\end{array}$ &.. & $1(<1 \%)$ \\
\hline Ischaemic cardiac pain &.. & $1(<1 \%)$ \\
\hline General pain &. & $1(<1 \%)$ \\
\hline Musculoskeletal back pain &. & $1(<1 \%)$ \\
\hline Musculoskeletal joint pain &. & \\
\hline Vascular thrombosis or embolism &. & $1 \%)$ \\
\hline
\end{tabular}

Data are $\mathrm{n}(\%)$. All grade 3 events are reported. Only those grades 1 and 2 events experienced by $10 \%$ or more of patients are reported. One $(<1 \%)$ patient had grade 4 cardiac ischaemia or infarction. No grade 5 adverse events were reported.

Table 2: Adverse events in the capecitabine group $(n=213)$ 


\begin{tabular}{|l|c|c|}
\hline & $\begin{array}{c}\text { Capecitabine group } \\
(\mathbf{n = 6 4 )}\end{array}$ & $\begin{array}{c}\text { Observation group } \\
(\mathbf{n = 2 9})\end{array}$ \\
\hline Category & & $29(100 \%)$ \\
\hline Unrelated serious adverse event & $31(48 \%)$ & 0 \\
\hline Serious adverse reaction & $33(52 \%)$ & $12(41 \%)$ \\
\hline Outcome & & $10(34 \%)$ \\
\hline Resolved, no sequelae & $55(86 \%)$ & $4(14 \%)$ \\
\hline Resolved, with sequelae & $6(9 \%)$ & $3(10 \%)$ \\
\hline Unresolved & $3(5 \%)$ & $\mathrm{NA}$ \\
\hline Death & 0 & $\mathrm{NA}$ \\
\hline Relatedness to treatment & & $\mathrm{NA}$ \\
\hline Unrelated & $23(36 \%)$ & $\mathrm{NA}$ \\
\hline Unlikely to be related & $14(22 \%)$ & $\mathrm{NA}$ \\
\hline Possibly related & $5(8 \%)$ & $\mathrm{NA}$ \\
\hline Probably related & $7(11 \%)$ & $\mathrm{NA}$ \\
\hline Definitely related & $15(23 \%)$ & $\mathrm{NA}$ \\
\hline Expectedness & & $51(80 \%)$ \\
\hline Expected & $5(8 \%)$ & \\
\hline Unexpected & $8(13 \%)$ & \\
\hline Missing data & & \\
\hline
\end{tabular}

Data are $\mathrm{n}(\%)$. Numbers are the frequency of events; patients might have more than one serious adverse event. NA=not applicable.

Table 3: Serious adverse events 


\begin{tabular}{|c|c|c|c|}
\hline & $\begin{array}{c}\text { Capecitabine group } \\
(n=223)\end{array}$ & $\begin{array}{c}\text { Observation group } \\
(n=224)\end{array}$ & $p$ value \\
\hline \multicolumn{4}{|l|}{ QLQ-LMC21 } \\
\hline Eating & $1.0(0.0-12.5)$ & $0.0(0.0-9.4)$ & $0 \cdot 18$ \\
\hline Pain & $11 \cdot 1(0 \cdot 0-26 \cdot 0)$ & $4.9(0.0-22 \cdot 2)$ & 0.061 \\
\hline Fatigue & $16 \cdot 7(0 \cdot 0-36 \cdot 1)$ & $10 \cdot 1(0 \cdot 0-31 \cdot 3)$ & 0.066 \\
\hline Social functioning & $3.5(0.0-15 \cdot 6)$ & $0.0(0.0-13 \cdot 9)$ & 0.11 \\
\hline Anxiety & $14 \cdot 3(0.0-36 \cdot 7)$ & $12 \cdot 5(0.0-30 \cdot 2)$ & 0.25 \\
\hline Weight loss & $0.0(0.0-9.4)$ & $0.0(0.0-6 \cdot 3)$ & $0 \cdot 11$ \\
\hline Taste & $0.0(0.0-11 \cdot 1)$ & $0.0(0.0-6 \cdot 3)$ & 0.042 \\
\hline Dry mouth & $0.0(0.0-16 \cdot 7)$ & $0.0(0.0-13.9)$ & 0.26 \\
\hline Sore mouth or tongue & $0.0(0.0-4 \cdot 2)$ & $0.0(0.0-4 \cdot 2)$ & 0.59 \\
\hline Peripheral neuropathy & $0.0(0.0-13.5)$ & $0.0(0.0-4.2)$ & 0.0016 \\
\hline Jaundice & $0.0(0.0-0.0)$ & $0.0(0.0-0.0)$ & 0.92 \\
\hline \multicolumn{4}{|l|}{ QLQ-C30 functioning scales } \\
\hline Physical & $82 \cdot 5(64 \cdot 0-92 \cdot 7)$ & $85 \cdot 0(70 \cdot 0-93 \cdot 3)$ & 0.16 \\
\hline Role & $72.9(50.5-91.7)$ & $81 \cdot 3(52 \cdot 8-91 \cdot 7)$ & 0.18 \\
\hline Emotional & $79.9(58 \cdot 9-92 \cdot 2)$ & $83 \cdot 3(64 \cdot 8-93 \cdot 2)$ & 0.36 \\
\hline Cognitive & $87.5(66 \cdot 1-96.4)$ & $87 \cdot 5(76 \cdot 0-100 \cdot 0)$ & 0.1 \\
\hline Social & $76 \cdot 2(56 \cdot 9-91 \cdot 7)$ & $83 \cdot 3(64 \cdot 6-95 \cdot 8)$ & 0.0060 \\
\hline Global health status or quality of life & $67.9(52 \cdot 1-80 \cdot 6)$ & $70 \cdot 8(56 \cdot 3-83 \cdot 3)$ & 0.18 \\
\hline \multicolumn{4}{|l|}{ QLQ-C30 symptoms scales } \\
\hline Fatique & $27 \cdot 8(15 \cdot 0-43 \cdot 3)$ & $27 \cdot 1(11 \cdot 1-38 \cdot 9)$ & 0.27 \\
\hline Nausea and vomiting & $2 \cdot 8(0.0-11.3)$ & $1.4(0.0-8.3)$ & 0.27 \\
\hline Pain & $17 \cdot 7(5 \cdot 2-38 \cdot 2)$ & $16 \cdot 7(6 \cdot 3-33 \cdot 3)$ & 0.8 \\
\hline Dyspnoea & $6 \cdot 3(0.0-25 \cdot 0)$ & $8 \cdot 3(0 \cdot 0-25 \cdot 0)$ & 0.43 \\
\hline Insomnia & $21 \cdot 9(4 \cdot 9-44 \cdot 1)$ & $20 \cdot 8(5 \cdot 6-41 \cdot 7)$ & 0.8 \\
\hline Appetite loss & $6 \cdot 3(0.0-18 \cdot 8)$ & $8 \cdot 3(0 \cdot 0-20 \cdot 8)$ & 0.88 \\
\hline Constipation & $4.2(0.0-22.9)$ & $2 \cdot 1(0 \cdot 0-16 \cdot 7)$ & 0.62 \\
\hline Diarrhoea & $8 \cdot 3(0.0-16 \cdot 7)$ & $4 \cdot 2(0 \cdot 0-16 \cdot 7)$ & 0.36 \\
\hline Financial difficulties & $2 \cdot 1(0 \cdot 0-22 \cdot 2)$ & $0.0(0.0-18 \cdot 8)$ & 0.35 \\
\hline
\end{tabular}

Data are median (IQR) standardised area under the curve unless otherwise specified. $p$ values were calculated from the Mann-Whitney test comparing the standardised area under the curve between treatment groups. All scales of the European Organisation for Research and Treatment of Cancer quality-of-life questionnaires QLQ-LMC21 and QLQ-C30 are shown.

Table 4: Patient-reported outcomes 